\title{
Continuous Wave MRI Diffusion Study of Water in Bentonite Clay
}

\author{
Andrew J. Fagan ${ }^{1,3}$, Nikolaus Nestle ${ }^{2}$, David J. Lurie ${ }^{1 *}$
}

${ }^{1}$ University of Aberdeen, Department of Bio-Medical Physics and Bio-Engineering, Foresterhill, Aberdeen AB25 2ZD, UK

2 TU Darmstadt, Institut für Festkörperphysik, Hochschulstraße 6, D-64289 Darmstadt

3 7T MR Facility, Wellcome Surgical Institute, University of Glasgow, Garscube Estate, Glasgow G61 1QH, UK.

* Corresponding author (telephone: 00441224 554061, email: lurie@biomed.abdn.ac.uk)

\begin{abstract}
Three-layered clay minerals such as montmorillonite (bentonite) exhibit very short transverse relaxation times. This is especially true for samples with relatively low water contents in the region of 20 to $30 \%$, which is the water content typically used in environmental technology applications (e.g. as a mineral liner material for landfills). The diffusion of water in samples with such short transverse relaxation times can be measured with NMR by observing the moisture gradients or isotope tracer fronts propagating through appropriately prepared samples by means of continuous wave (CW) MRI. The first results from such studies on bentonite clays are presented in this paper.
\end{abstract}

Keywords: NMR, clay, mineral liner, diffusion, CW-MRI

\section{Introduction}

Diffusion in clays was successfully studied by field gradient NMR techniques for high water-content clay gels and for two-layered, non-swellable clay materials such as kaolinite [1, 2]. At low water contents, water in non-swelling clays such as kaolinite still exhibits sufficiently long transverse relaxation times for field gradient NMR experiments. By contrast, the transverse relaxation times in three-layered clay minerals such as bentonite are decreased to a greater extent compared to the two-layered systems, a feature which is already evident at high water contents. Bentonite gels with a high water content have therefore been investigated in medical MRI as a negative gastrointestinal contrast agent [3]. The relaxation is further enhanced by the presence of some paramagnetic impurities which are invariably found in "white" technically pure bentonite.

In addition to field gradient diffusometry, NMR also offers a second option for diffusometry: the observation of the temporal evolution of substance profiles by MRI. With a sufficiently symmetrical sample design, onedimensional profiles obtained from MRI are sufficient for determining the diffusion coefficient of the substance of interest. In contrast to field-gradient NMR, it is not possible to observe pure self-diffusion in such an approach. Therefore, one is constrained to study either diffusion processes along a moisture gradient or the propagation of a stable isotope tracer profile (i.e. the diffusive mixing between ordinary water and 
deuterium oxide in the case of water self-diffusion in clay). Furthermore, since conventional MRI suffers even more than field gradient diffusometry from the short transverse relaxation times in low-water content bentonite clays, one must apply specialized MRI techniques whose performance is not affected strongly by the short relaxation times. Possible options are STRAFI [4], SPI [5] and CW-MRI [6]. Since no dead-times need to be considered in CW-MRI, this technique is the one least affected by short relaxation times of all the possible approaches.

\section{Materials and Methods}

Continuous wave MRI experiments were carried out using the home-built $7 \mathrm{~T}$ imaging system at the Department of Bio-Medical Physics and Bio-Engineering, University of Aberdeen [6]. Clay samples were prepared in PTFE containers with an inner diameter of $30 \mathrm{~mm}$ and a length of about $50 \mathrm{~mm}$. Laboratory grade kaolinite and bentonite powder was obtained from Sigma-Aldrich (Poole, UK) and mixed in powder form with the appropriate quantities of water or heavy water. After mixing, the moistened powder was filled in several layers into the sample container. Each layer was condensed into a homogeneous clay mass by heavily stamping with a rounded metal bar for several minutes. By this technique both thick monolayers of clay and multilayers samples with different water contents or from different types of clay could be produced.

\section{Results and Discussion}

In this contribution, we restrict ourselves to reporting results from diffusion experiments in pure bentonite samples: One series of experiments was conducted on samples consisting of a homogeneous bentonite layer with a water content of $20 \%$ in weight which was brought into contact with a reservoir of laboratory tissue soaked with several $\mathrm{ml}$ of water. This corresponds to an interdiffusion scenario along a moisture gradient. In another type of sample, the self-diffusion of water between two bentonite layers prepared with light and heavy water was studied. The water content in this experiment was chosen to be $30 \%$ in weight for the light water and $33.3 \%$ in weight for the heavy water. The results of these experiments are presented in figure (1). The profiles in figure (1a) are somewhat disturbed by the presence of a chemical shift artifact at the interface between the water reservoir and the bentonite sample (illustrated in more detail in figure (2)). Nevertheless, both for diffusive moisture transport and for self-diffusion of light water into heavy water, the analysis of the diffusion front as a function of time shows in both cases a nice $\mathrm{t}^{2}$-dependence of the front line which indicates Fickian diffusion and allows for the determination of a diffusion coefficient according to $x^{2}=2 D t$ from the slope of the curve (see figure 3). The resulting value for the self-diffusion coefficient of $[8.4 \pm 0.5] \times 10^{-10} \mathrm{~m}^{2} / \mathrm{s}$ is the same order of magnitude as that recorded for non-swelling technical-grade kaolinite at similar water content [2]. This indicates that the difference in molecular dynamics between bentonite and kaolinite clays (which is quite obvious from the different NMR relaxation behaviour of both materials) does not lead to a strong effect on the long-range diffusive transport behaviour of the water molecules and that the tortuosities encountered by the molecules are similar. 


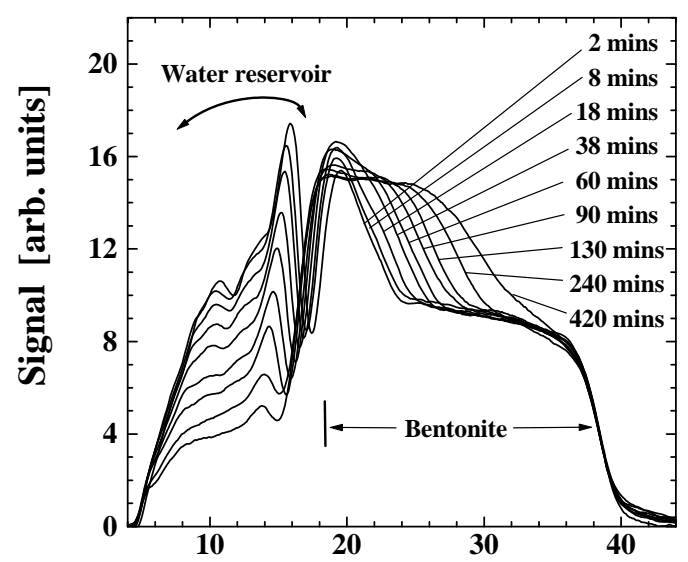

(a)

Distance [mm]

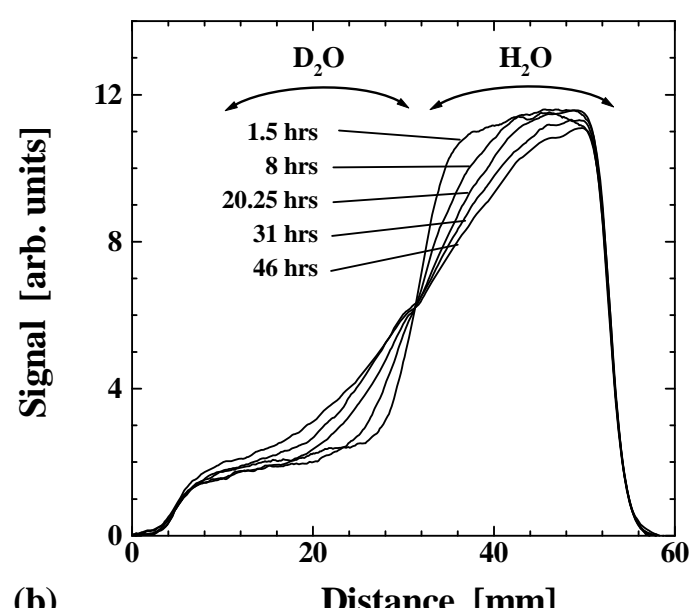

(b)

Distance [mm]

Figure 1: Diffusion profiles of water into bentonite. (a) Diffusion of water from a reservoir (left hand side) into bentonite clay with initially $20 \% \mathrm{w} / \mathrm{b}$ water. At the interface between the bentonite and the water reservoir, a chemical shift artifact is observed (illustrated in more detail in figure 2). (b) Diffusion of water between two bentonite clay layers initially prepared with heavy water (left hand side) and light water (right hand side).

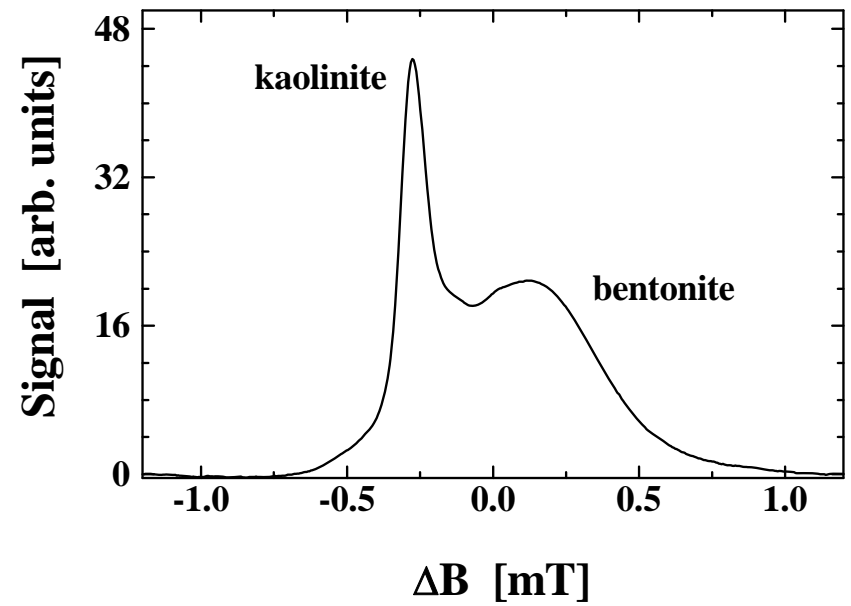

Figure 2: Shift in resonance lines between a kaolinite layer (narrow line, no notable shift compared to water) and a bentonite layer. The shift corresponds to about 56 ppm and thus indicates the presence of paramagnetic impurities in the bentonite. 


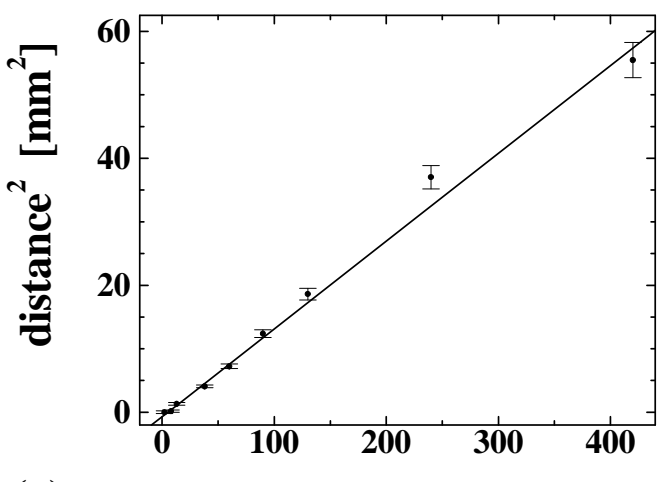

(a)

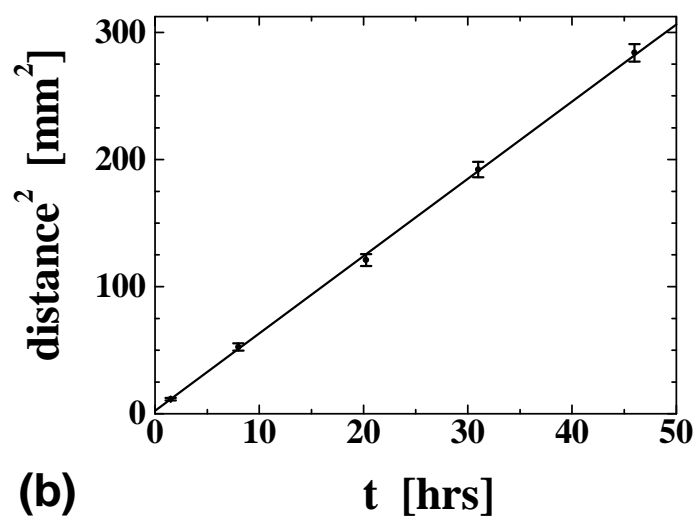

Figure 3: Determination of the diffusion coefficients from the observed diffusion fronts shown in figure 1:

(a) Diffusion along moisture gradient. The resulting diffusion coefficient was found to be [1.15 \pm 0.05$] \times 10^{-}$ ${ }^{9} \mathrm{~m}^{2} / \mathrm{s}$, (b) Self-diffusion scenario for $30 \% \mathrm{w} / \mathrm{b}$ moisture content. The diffusion coefficient was determined as $[8.56 \pm 0.1] \times 10^{-10} \mathrm{~m}^{2} / \mathrm{s}$

\section{References}

1 Nakashima Y. 2000 Effects of clay fraction and temperature on the $\mathrm{H}_{2} \mathrm{O}$ self-diffusivity in hectorite gel: A pulsed-field-gradient spin-echo nuclear magnetic resonance study. Clay Clay Miner, 48, 603-609.

2 Nestle N. NMR studies of transport and remediation processes in soil and sediments. In: Lens, P editor. Integrated Environmental Technology Series, IWA Publishing, London, 2005, in press.

3 Paley M R, Nicolas AI, Mergo PJ, Torres GM, Burton SS, Pos PR 1997 Low density barium and bentonite mixture versus high-density barium: a comparative study to optimize negative gastrointestinal contrast agents Magn Reson Imaging 15 1033-1036.

4 Randall EW, Mahieu N, Ivanova GI 1997 NMR studies of soil, soil organic matter and nutrients: spectroscopy and imaging Geoderma 80 307-325

5 Beyea SD, Balcom BJ, Mastikhin IV, Bremner TW, Armstrong RL, Grattan-Bellew PE 1999 Imaging of Heterogeneous Materials with a Turbo Spin Echo Single Point Imaging Technique J Magn Reson 144 255-265

6 Fagan AJ, Davies GR, Hutchison JMS, Lurie DJ 2003 Continuous wave MRI of heterogeneous materials J Magn Reson 163 318-324. 